\title{
Configurações
}

Revista de sociologia

\section{La difusión de los valores expresivos en el trabajo}

Diffusion of expressive work values

Distribuition des valeurs expressives du travail

José Luis Veira Veira y Celia Muñoz Goy

\section{CpenEdition}

\section{Journals}

Edición electrónica

URL: http://journals.openedition.org/configuracoes/168

DOI: $10.4000 /$ configuracoes. 168

ISSN: 2182-7419

\section{Editor}

Centro de Investigação em Ciências Sociais

\section{Edición impresa}

Fecha de publicación: 30 junio 2010

Paginación: 13-30

ISSN: 1646-5075

\section{Referencia electrónica}

José Luis Veira Veira y Celia Muñoz Goy, «La difusión de los valores expresivos en el trabajo », Configurações [En línea], 7 | 2010, Puesto en línea el 18 febrero 2012, consultado el 30 abril 2019. URL http://journals.openedition.org/configuracoes/168; DOI : 10.4000/configuracoes. 168

Este documento fue generado automáticamente el 30 abril 2019.

(c) CICS 


\title{
La difusión de los valores expresivos en el trabajo
}

\author{
Diffusion of expressive work values \\ Distribuition des valeurs expressives du travail
}

José Luis Veira Veira y Celia Muñoz Goy

\section{Introducción: Las dos caras del trabajo}

1 El trabajo, como el dios Jano, tiene dos caras, una hosca y otra agradable. Pero ambas forman una unidad. Las distintas teorías acerca del trabajo suelen distinguirse por el énfasis puesto en una de las dos caras. La cara hosca se presenta cuando se considera el trabajo como una actividad que ha de hacerse forzadamente para subsistir y que no se haría en caso de no darse este carácter obligatorio. Marx calificaba el trabajo bajo las condiciones capitalistas como "trabajo forzado", pues el trabajador no tiene más alternativa que vender su fuerza de trabajo si quiere subsistir. Desde esta perspectiva el trabajo aparece como una explotación injusta (curiosamente coincidente con la maldición divina

2 de la Biblia: ganarás el pan con el sudor de tu frente). En este caso el trabajo no se contempla como un fi $\mathrm{n}$ en sí mismo sino como un medio para obtener algún tipo de recompensa material que alivie el sufrimiento derivado del esfuerzo.

3 Esta perspectiva descansa en la creencia ampliamente compartida de que el trabajo representa una relación mercantil desigual en la que unos (los empleadores) compran trabajo y otros (los empleados) venden su esfuerzo. La venta de este esfuerzo se lleva a cabo para satisfacer dos de las necesidades más básicas en la escala de Maslow (1954): la supervivencia y la seguridad. De ahí que la orientación instrumental hacia el trabajo sea una de las actitudes más popularmente extendida (se trabaja para vivir y no se vive para trabajar).

4 Sin embargo también existe la cara amable del trabajo. El desarrollo del capitalismo propició tres tipos de creencias al respecto que confirieron al trabajo un significado muy 
distinto al de castigo divino o de explotación. La primera de ellas es la que considera el trabajo como un bien común por ser fuente de riqueza y poder para las naciones (creencia defendida por la mayoría de los padres fundadores de la economía); la segunda es la que atribuye al trabajo un significado moral, convirtiéndolo en una obligación social (idea mantenida por algunas religiones, por ejemplo por la ética calvinista impulsora del desarrollo económico capitalista, para conjurar los peligros que se derivan de un ocio prolongado) y finalmente una tercera creencia es la consideración del trabajo como una actividad que permite la auto-realización de la persona mediante el despliegue de todas sus capacidades. Los tres tipos de creencias tienen en común que superan la visión meramente mercantil del trabajo, trascendiéndola y propiciando así las actitudes expresivas hacia el trabajo: el trabajo es algo más que mera supervivencia, también puede contribuir a una existencia más feliz y al desarrollo pleno de la personalidad.

5 Ambas caras del trabajo son reales aunque las creencias que las sustentan se distribuyen de forma irregular entre los individuos, las sociedades y las culturas. La cara hosca del trabajo favorece el desarrollo de actitudes instrumentales

6 o "materialistas", mientras que la cara amable propicia orientaciones "autoexpresivas" o de auto-realización. Las actitudes instrumentales se orientan a la satisfacción de necesidades materiales poniendo énfasis en un buen salario o en la estabilidad del empleo; las actitudes "expresivas" por el contrario buscan la satisfacción de necesidades superiores en la escala de Maslow, como pueden ser las relaciones personales y el crecimiento personal en una tarea interesante y que presente retos.

\section{Las orientaciones laborales}

7 En la extensa literatura sobre los modos de valorar las funciones y recompensas del trabajo (Goldthorpe et al., 1968; Cherrington, 1980; Yankelovich, 1985, 1994; Inglehart, 1991; Zanders, 1994; MOW, 1987; Russell, 1998; Ros, Schwartz \& Surkiss, 1999) pueden apreciarse diferentes clasificaciones de las orientaciones laborales, pero, en todas ellas, hay alguna orientación o eje que se refiere a una visión instrumental o extrínseca del trabajo y alguna orientación o eje que se refi ere a una visión expresiva o intrínseca.

La orientación instrumental hace referencia a una visión del trabajo que valora las recompensas materiales y económicas del trabajo, especialmente el salario, y otro tipo de recompensas que no tienen por qué ser exclusivamente económicas, como puede ser la estabilidad del puesto de trabajo. Por otra parte, la orientación expresiva se refiere a la valoración de las recompensas intrínsecas al propio desempeño de la tarea, que posibilitan el desarrollo y la realización personal y, además, aquellas características del trabajo que permiten la interacción social de los trabajadores.

9 La tendencia a situar las orientaciones instrumentales y expresivas del trabajo como contrapuestas deriva, en numerosas ocasiones, de la forma en que se miden los aspectos relativos al trabajo. Muchas veces se realiza una elección entre distintos resultados o recompensas del trabajo, lo que puede llevar a pensar que los que prefieren las recompensas de tipo expresivo es porque no perciben que las recompensas instrumentales sean necesarias o importantes. Sin embargo, esta interpretación no sería correcta pues en realidad lo que se estaría apreciando es que dicha persona antepone las recompensas expresivas a las instrumentales, aunque estas también puedan ser importantes, es decir que, a la hora de elegir, antepone al salario o la estabilidad otras 
recompensas, como pueden ser las condiciones laborales que le permitan desarrollar su potencial personal, que le planteen retos y que, en definitiva, contribuyan a mejorar su calidad de vida en el trabajo.

10 En el análisis de las orientaciones hacia el trabajo y su posible evolución existen dos enfoques diferentes. Por una parte, puede suponerse que el subsistema social económico es, por definición, el ámbito de la escasez (Goldthorpe et al., 1968) y por esta razón cabría esperar que en el mundo laboral se deje notar la influencia de los valores fuertemente asociados a las necesidades básicas de supervivencia y seguridad, es decir, valores de tipo materialista que se corresponderían con un puesto de trabajo estable, seguro y con un buen sueldo, o lo que es lo mismo, que en las orientaciones laborales siempre habrá un componente instrumental subyacente.

11 Por otra parte, algunos autores (Inglehart, 1991, 1998; Yankelovich, 1985; Zanders, 1994; Harding \& Hikspoors, 1995; Russell, 1998; Veira Veira y Muñoz Goy, 2004) señalan que en el ámbito laboral, a nivel agregado, se está produciendo un cambio de valores acorde con el experimentado en el ámbito general, por lo que se espera un auge de los aspectos vinculados a las necesidades de tipo secundario ${ }^{1}$ como la autorrealización, la afiliación o el espíritu de logro, esto es, valores de tipo posmaterialista, que en el ámbito del trabajo se refl ejarían en orientaciones de tipo expresivo.

12 Ambas perspectivas han tratado de dilucidar qué tipo de orientación laboral (instrumental o expresiva) es la prevaleciente y cuál es la dirección del cambio de valores. El estudio llevado a cabo por Goldthorpe mostró algo muy importante: que el desarrollo de la sociedad de consumo había destruido la ética calvinista del trabajo y convertido éste en un medio instrumental para contribuir al mantenimiento y bienestar familiar de los trabajadores. Esta perspectiva refuerza la hipótesis de que prevalecerán las orientaciones instrumentales sobre las expresivas, siempre y cuando se mantengan las actuales condiciones de la sociedad de consumo.

13 La otra perspectiva supone que la tendencia en general es hacia una prevalencia de los valores expresivos en la medida que se logre una prolongada estabilidad política y un desarrollo económico. Los protagonistas de este cambio son las distintas cohortes que crecieron en épocas de prosperidad y estabilidad que no solo tratarán de vivir acordes con los nuevos valores posmaterialistas (que en el ámbito laboral se corresponderían con actitudes expresivas) sino que además los transmitirán a las generaciones futuras. Esta teoría del cambio generacional presenta, sin embargo, algunos problemas cuando se aplica directamente al mundo concreto de las orientaciones laborales y a cuyo esclarecimiento quiere contribuir el presente artículo.

\section{Proposiciones teóricas relevantes}

14 Algunos autores (Inglehart, 1991, 1998; Inglehart \& Welzel, 2006; Abramson \& Inglehart, 1992; Yankelovich, 198, 1994) sostienen que el reemplazo generacional es el mecanismo por el que se produce el cambio en los valores en las sociedades. Desde esta perspectiva, que propone que la evolución de los valores laborales seguirá la misma dirección que los valores en el ámbito general, la razón de que los nuevos valores se encuentren más extendidos entre los más jóvenes se debería a que estos han sido socializados en épocas de mayor estabilidad y mejores condiciones económicas, lo que les permite dar por supuesta la cobertura de las necesidades primarias, y por tanto cabría esperar que presenten una 
mayor tendencia hacia las orientaciones laborales expresivas. Por ello, la cohorte por año de nacimiento será una variable importante a la hora de describir las opiniones sobre los valores laborales y explicar o pronosticar la dirección del cambio.

Como se ha señalado más arriba, desde un punto de vista diferente, Goldthorpe et al. (1968), MOW (1987) y Sverko y Super (1995) señalan que las diferencias en los valores laborales de los distintos grupos de edad se deben a la posición que la persona ocupa en el ciclo vital, por lo que no cabe esperar que las cohortes por año de nacimiento mantengan los mismos valores laborales a lo largo de toda la vida, lo que entraría en contradicción con la explicación de la evolución de valores por el reemplazo generacional.

En el debate sobre los determinantes de la evolución de los valores a nivel general - paso del materialismo al posmaterialismo - existe aún otro enfoque, propuesto por Duch y Taylor $(1993,1994)$, que sostiene que la educación constituye una importante variable explicativa, puesto que un elemento integral del posmaterialismo es el apoyo a los valores democráticos que, precisamente, se encuentran relacionados con la educación en los niveles más elevados.

17 La explicación de esta relación puede consistir tanto en el hecho de que la educación imparta y refuerce valores liberales como la igualdad, la tolerancia y el respeto por la libertad individual, como en que las personas con niveles educativos altos tengan más probabilidades de verse expuestas o de ser socializadas en las normas sociales que promueven los valores democráticos.

18 Según Duch y Taylor (1993: 755-756) cualquiera de estas dos explicaciones sirve para afirmar que las cohortes que han alcanzado niveles educativos más altos son las que presentan porcentajes más altos de valores posmaterialistas, es decir que los efectos de cohorte que Inglehart atribuye a los crecientes niveles de riqueza son simplemente el resultado de los crecientes niveles de educación.

19 Si se lleva esta controversia al terreno de los valores laborales cabría preguntarse si efectivamente es la generación o el nivel de estudios la variable que mejor ayuda a explicar las orientaciones hacia el trabajo, teniendo en cuenta, además, que en diversos estudios - Yankelovich (1985), Zanders (1994) y Russell (1998) - se han encontrado claras relaciones entre dicha variable y los valores laborales, especialmente en el sentido de que las personas con mayores niveles de estudios suelen orientarse con mayor frecuencia hacia los valores laborales expresivos.

La influencia del nivel de estudios sobre las orientaciones laborales podría explicarse por el hecho de que los más educados son, precisamente, los que pueden permitirse aspirar a unos puestos de trabajo más acordes con las exigencias de la autorrealización. Asimismo, es posible que al recibir una educación formal prolongada se "aprenda" que es deseable la autorrealización y, además, que se podría alcanzar en el ámbito del trabajo.

21 Para resumir, puede decirse que existen dos proposiciones teóricas alternativas para la explicación de la distribución y difusión de las orientaciones expresivas hacia el trabajo. La primera de ellas pone énfasis en el cambio generacional, suponiendo que existe una fuerte correlación entre las distintas cohortes de edad y las orientaciones expresivas en el sentido de que éstas serán más frecuentes entre las cohortes de menor edad que entre las de mayor edad.

22 La segunda proposición teórica centra su atención sin embargo en la importancia del nivel de estudios como factor determinante de las actitudes hacia el trabajo, afirmando que las orientaciones expresivas serán más frecuentes entre las personas con mayor nivel 
de estudios. Como es sabido, la variable nivel de estudios es una de las tres dimensiones más importante, junto con el nivel de ingresos y la ocupación, para determinar el status socioeconómico del individuo. Por esta razón, al analizar el impacto del nivel de estudios en las orientaciones laborales habrán de tenerse en cuenta también otros aspectos del status de la persona que pudieran estar contaminando el efecto del nivel de estudios, como son los ingresos, la satisfacción con la situación financiera del hogar, la ocupación, la edad, la situación laboral y el sexo.

Autores como Yankelovich $(1985,1994)$, Inglehart $(1991,1998)$ y Rokeach $(1973)$ señalan que entre las personas que se encuentran en mejor situación económica cabría esperar una orientación más expresiva, pues, al tener sus necesidades básicas cubiertas, pueden verse libres para aspirar a una experiencia laboral creativa, enriquecedora e interesante. Para dar cuenta de la situación económica a nivel individual se han considerado las variables que recogen el nivel de ingresos del hogar y el grado de satisfacción con la situación económica del hogar.

Las diferencias por género en los valores laborales se pueden agrupar, como señalan Rowe y Snizek (1995), en dos enfoques: el modelo de la socialización y el modelo estructural. El modelo de la socialización sostiene que las diferencias observadas en los valores laborales reflejan los patrones tradicionales asignados a cada género, mientras que, por el contrario, el modelo estructural afi rma que tales diferencias son un reflejo del acceso diferencial de varones y mujeres al sistema de recompensas laborales y a las posiciones jerárquicas. A pesar de que en distintos estudios sobre el tema (Dex, 1988; Vaus y McAllister, 1991; Rowe y Snizek, 1995; Abu-Saad, 1997) se concluye que los estereotipos sobre las orientaciones hacia el trabajo de varones y mujeres resultan inapropiados, bien porque las diferencias encontradas son muy escasas o bien porque, en caso de existir, se debilitan al establecer controles por distintas variables relacionadas con el modelo de la socialización o, sobre todo, con el modelo estructural, se explorará la relación del género con las orientaciones laborales, para comprobar si existen diferencias en los países analizados.

Por último, en diversas investigaciones - Pino y Bericat, 1996; Ayerbe, 2000; Muñoz Goy, 2005 - se ha encontrado que la situación laboral permite establecer diferencias en las actitudes hacia el trabajo. Dichas diferencias pueden deberse a las distintas posiciones que el trabajo ocupa en la vida -lo que llevaría a establecer una diferencia entre la población activa y el resto-, o como consecuencia de la relación que tiene la situación laboral con la edad y esta con el momento en el ciclo vital de las personas.

En cuanto a la ocupación ya se ha señalado que es una variable determinante del status socioeconómico del individuo, junto con el nivel de estudios e ingresos y por esta razón debe siempre estar controlada si se desea obtener el impacto del nivel de estudios independientemente de la ocupación.

El análisis que sigue a continuación trata de verificar ambas proposiciones teóricas con el fin de mejorar la comprensión y explicación de los determinantes del cambio de valores en el ámbito del trabajo. Los países incluidos en la muestra pertenecen a Europa y se encuentran entre las sociedades más ricas del mundo. Por esta razón cabe esperar que exista una gran difusión de las actitudes expresivas entre sus respectivas poblaciones, lo cual permitirá aclarar mejor los determinantes de su expansión, distribución y difusión. Aunque los datos tienen un origen individual no por ello se excluye el análisis comparado a nivel macro, esto es, por países. 


\section{Bases de datos y metodología} arriba. Puesto que en la mayor parte de los casos (nivel de estudios, cohorte por año de nacimiento, ocupación, nivel de ingresos y satisfación con la situación financiera del hogar) se pronostica una dirección en la relación de las variables se ha utilizado como medida de asociación el coefi ciente de correlación Rho de Spearman para variables ordinales, que permite conocer no sólo la intensidad de la asociación sino también el sentido de la misma. En aquellos casos en los que no se puede establecer la dirección en al menos una de las variables que se incluyen en la relación (situación laboral, sexo y país de realización de la encuesta) se ha optado por calcular el coeficiente $\mathrm{V}$ de Cramer, que únicamente indica la intensidad de la asociación. 


\section{Análisis de datos y resultados}

Los resultados muestran (tabla 1), en primer lugar, que existe una clara relación entre las orientaciones laborales y el nivel de estudios en la dirección esperada, es decir que entre los entrevistados con mayores niveles educativos hay una mayor demanda de recompensas laborales de tipo expresivo.

También se observan asociaciones positivas, aunque menos intensas que la anterior, entre las orientaciones laborales y las variables relacionadas con el ámbito económico como son el nivel de ingresos y la satisfacción con la situación fi nanciera del hogar, es decir que las personas con mayores ingresos o más satisfechas con su economía doméstica tienden a acentuar más los aspectos expresivos del trabajo.

En cuanto a la ocupación, el signo negativo de la correlación corresponde al hecho de que las ocupaciones de mayor nivel se han codifi cado con números inferiores que las de menor nivel, por lo que la correlación debe interpretarse como que a medida que nos dirigimos a las ocupaciones de mayor nivel de capacitación o que ocupan posiciones más elevadas en la jerarquía laboral aumentan los porcentajes de personas con orientaciones expresivas.

Sin embargo, no se han encontrado relaciones estadísticamente signifi cati-vas entre las orientaciones laborales y las cohortes por año de nacimiento, por lo que no se puede concluir que entre los más jóvenes haya porcentajes de orientaciones expresivas más elevados que entre las personas de mayor edad ${ }^{3}$.

Tabla 1: Correlaciones entre las orientaciones laborales y distintas variables explicativas ${ }^{a}$ para una muestra de diez países europeos

\begin{tabular}{|c|c|c|c|c|c|c|c|c|}
\hline & $\begin{array}{l}\text { Oriontacioner } \\
\text { laborsbi }\end{array}$ & $\begin{array}{l}\text { Hinclde } \\
\text { artadior }\end{array}$ & 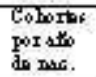 & Qctursion & $\begin{array}{l}\text { Ecish } \\
\text { do } \\
\text { ingorof }\end{array}$ & 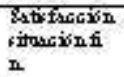 & $\begin{array}{l}\text { Sinotion } \\
\text { laborsl }\end{array}$ & $20 \times 0$ \\
\hline $\begin{array}{l}\text { Hinclde } \\
\text { urtoliof }\end{array}$ & $\begin{array}{l}0,286++0,000 \\
11210\end{array}$ & & & & & & & \\
\hline 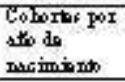 & $\begin{array}{l}0,0030,745 \\
11294\end{array}$ & $\begin{array}{l}0,147^{6+} \\
0,000 \\
11514\end{array}$ & & & & & & \\
\hline Qcistion & $\begin{array}{l}-0,091+{ }^{0}, 000 \\
10909\end{array}$ & $\begin{array}{l}-0,305+4 \\
0,000 \\
11022\end{array}$ & $\begin{array}{l}0,027^{6} \\
0,020 \\
11106\end{array}$ & & & & & \\
\hline $\begin{array}{l}\text { Erasle de } \\
\text { ingrerof }\end{array}$ & $\begin{array}{l}0,135 \times+0,000 \\
9783\end{array}$ & $\begin{array}{l}0,393^{\circ+6} \\
0,000 \\
9961\end{array}$ & $\begin{array}{l}0,06{ }^{6} \\
0,000 \\
10017\end{array}$ & $\begin{array}{l}-0,15366 \\
0,0009685\end{array}$ & & & & \\
\hline 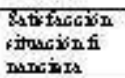 & $\begin{array}{l}0,188^{+}+0,000 \\
12113\end{array}$ & $\begin{array}{l}0,185^{\circ 6} \\
0,000 \\
11+36\end{array}$ & $\begin{array}{l}-0,10606 \\
0,000 \\
11520\end{array}$ & $\begin{array}{l}-0,090+6 \\
0,000 \\
11024\end{array}$ & $\begin{array}{l}0,379+4 \\
0,000 \\
9974\end{array}$ & & & \\
\hline $\begin{array}{l}\text { Siroucion } \\
\text { hlorisl }\end{array}$ & $\begin{array}{l}0,077^{+60,000} \\
11276\end{array}$ & $\begin{array}{l}0,16306 \\
0,000 \\
11+94\end{array}$ & $\begin{array}{l}0,39564 \\
0,000 \\
11573\end{array}$ & $\begin{array}{l}0,276 \% \\
0,000 \\
11085\end{array}$ & $\begin{array}{l}0,1+3 \times 6 \\
0,000 \\
10034\end{array}$ & $\begin{array}{l}0,108+6 \\
0,00011+96\end{array}$ & & \\
\hline $80 \times 0$ & $\begin{array}{l}0,089+0,005 \\
113+3\end{array}$ & $\begin{array}{l}0,0+8+4 \\
0,000 \\
11555\end{array}$ & $\begin{array}{l}0,0+5 \% \\
0,001 \\
11651\end{array}$ & $\begin{array}{l}0,256 \% \\
0,000 \\
111+8\end{array}$ & $\begin{array}{l}0,079+4 \\
0,000 \\
10078\end{array}$ & $\begin{array}{l}0,00556 \\
0,00511565\end{array}$ & $\begin{array}{l}0,352^{+6} \\
0,000 \\
11575\end{array}$ & \\
\hline Pas & $\begin{array}{l}0,238+60,000 \\
113+3\end{array}$ & $\begin{array}{l}0,256 \%+ \\
0,000 \\
11553\end{array}$ & $\begin{array}{l}0,078 \% 4 \\
0,000 \\
11649\end{array}$ & $\begin{array}{l}0,327+4 \\
0,000 \\
111+8\end{array}$ & $\begin{array}{l}0,183+4 \\
0,000 \\
10074\end{array}$ & $\begin{array}{l}0,125+4 \\
0,00011562\end{array}$ & $\begin{array}{l}0,1+0 \% 4 \\
0,000 \\
11575\end{array}$ & $\begin{array}{l}0,026 \\
0,566 \\
11651\end{array}$ \\
\hline
\end{tabular}

Fuente: ASEP, Encuesta Mundial de Valores 2005-2008. Elaboración propia.

a Las correlaciones mostradas son el coeficiente Rho de Spearman, excepto para las relaciones con las variables situación laboral, sexo y país, en cuyos casos se muestran el coeficiente $V$ de Cramer En cada casilla de la tabla se muestra en primer lugar la correlación, en segundo lugar el nivel de signifı cación y en tercer lugar el tamaño de la muestra (excluidos los casos de no respuesta). ** La correlación es signifi cativa al nivel 0.01 (bilateral)

* La correlación es signifi cativa al nivel 0.05 (bilateral)

40 Para las variables situación laboral, sexo y país, se han encontrado asociaciones signifi cativas con las orientaciones laborales en todos los casos, pero, al tratarse de variables 
nominales, la relación debe interpretarse a la vista de las tablas de contingencia, que se presentan a continuación.

41 En cuanto al sexo (tabla 2), se aprecia que las mujeres tienen una mayor tendencia hacia las opciones expresivas que los varones. Tal como se señaló más arriba, varios estudios coinciden en atribuir a las mujeres una mayor propensión a la demanda de recompensas expresivas en el trabajo, aunque por distintas razones según las diferentes aproximaciones teóricas.

Tabla 2: Orientaciones laborales por sexo

\begin{tabular}{|c|c|c|c|c|c|}
\hline \multicolumn{7}{|c|}{ Orientaciones Laborales } \\
\hline Sexo & Instrumental & $\begin{array}{c}\text { Mixto- } \\
\text { Instrumental }\end{array}$ & $\begin{array}{c}\text { Mixto- } \\
\text { Expresivo }\end{array}$ & Expresivo & Total \\
\hline Varones & $25,9 \%$ & $30,0 \%$ & $25,7 \%$ & $18,5 \%$ & $100,0 \%(\mathrm{n}=5489)$ \\
\hline Mujeres & $20,3 \%$ & $28,3 \%$ & $26,8 \%$ & $24,6 \%$ & $100,0 \%(\mathrm{n}=5854)$ \\
\hline Total & $23,0 \%$ & $29,1 \%$ & $26,3 \%$ & $21,6 \%$ & $100,0 \%(\mathrm{n}=11343)$ \\
\hline
\end{tabular}

Fuente: ASEP, Encuesta Mundial de Valores 2005-2008. Elaboración propia.

Según la situación laboral (tabla 3), los empleados a tiempo parcial, los autónomos y los estudiantes se inclinan más hacia posiciones expresivas o mixtoexpresivas, mientras que las amas de casa y los desempleados tienden más a las posiciones instrumentales o mixtoinstrumental. Los empleados a tiempo completo y, en menor medida, los jubilados se comportan de un modo similar al total, en el que se aprecia una distribución casi uniforme en las orientaciones laborales.

Tabla 3: Orientaciones laborales por situación laboral

\begin{tabular}{|c|c|c|c|c|c|}
\hline \multicolumn{6}{|c|}{ Orientaciones Laborales } \\
\hline Sinación Laboral & Instrumental & $\begin{array}{c}\text { Mristo- } \\
\text { Instrumentsl }\end{array}$ & $\begin{array}{l}\text { MGínto- } \\
\text { Expresino }\end{array}$ & Expresino & Total \\
\hline $\begin{array}{c}\text { Trobajudores a tiempo } \\
\text { completo }\end{array}$ & $22,7 \%$ & $29,5 \%$ & $27,4 \%$ & $20.4 \%$ & $100,0 \%(n=4617)$ \\
\hline $\begin{array}{c}\text { Trabajadores a tiempo } \\
\text { parcinl }\end{array}$ & $17,4 \%$ & $269 \%$ & $26.8 \%$ & $28.9 \%$ & $100,0 \%(\mathrm{n}=1251)$ \\
\hline Solitoromos & $195 \%$ & $24,7 \%$ & $298 \%$ & $260 \%$ & $100,0 \%(n=795)$ \\
\hline Jubilados/Pensionistos & $24,0 \%$ & $313 \%$ & $24,4 \%$ & $203 \%$ & $1000 \%(n=2467)$ \\
\hline Ama de casa & $330 \%$ & $29,1 \%$ & $21,1 \%$ & $168 \%$ & $100,0 \%(n=736)$ \\
\hline Estudiartes & $179 \%$ & $24,0 \%$ & $31,7 \%$ & $26,5 \%$ & $100,0 \%(n=654)$ \\
\hline Pardos & $30.8 \%$ & $33,2 \%$ & $19,2 \%$ & $168 \%$ & $100,0 \%(n=578)$ \\
\hline Otros & $225 \%$ & $275 \%$ & $315 \%$ & $185 \%$ & $100,0 \%(n=178)$ \\
\hline Total & $23,0 \%$ & $29,1 \%$ & $263 \%$ & $21.6 \%$ & $100,0 \%(n=11276)$ \\
\hline
\end{tabular}

Fuente: ASEP, Encuesta Mundial de Valores 2005-2008. Elaboración propia.

En cuanto al país de realización de la encuesta, los mayores porcentajes de personas expresivas se encuentran en Suiza y Suecia, donde más del $40 \%$ de la muestra eligen opciones expresivas puras y más de un $30 \%$ se sitúan en opciones mixtas-expresivas. A continuación aparecen Noruega y Holanda, donde las elecciones de posiciones de tipo expresivo y mixto-expresivo superan a la media. En el extremo opuesto destacan Alemania y especialmente España con porcentajes de orientaciones instrumentales que superan con creces a la media de los países analizados. 
Tabla 4: Orientaciones laborales por países

\begin{tabular}{|c|c|c|c|c|c|}
\hline \multicolumn{6}{|c|}{ Orientaciones laborales } \\
\hline & Instrumertal & $\begin{array}{c}\text { Mrixto- } \\
\text { Instrumertsl }\end{array}$ & $\begin{array}{c}\text { Mristo- } \\
\text { Expresino }\end{array}$ & Expresino & Totsl \\
\hline Francin & $25,2 \%$ & $32,7 \%$ & $243 \%$ & $179 \%$ & $1000 \%(\mathrm{n}=977)$ \\
\hline Gran Bretring & $20,1 \%$ & $323 \%$ & $30,2 \%$ & $17,4 \%$ & $1000 \%(n=987)$ \\
\hline Italin & $275 \%$ & $329 \%$ & $26,6 \%$ & $130 \%$ & $1000 \%(n=992)$ \\
\hline Holmin & $153 \%$ & $286 \%$ & $315 \%$ & $24,6 \%$ & $1000 \%(n=993)$ \\
\hline Esparin & $470 \%$ & $23,6 \%$ & $22,1 \%$ & $73 \%$ & $1000 \%(\mathrm{r}=1185)$ \\
\hline Horuega & $10,7 \%$ & $276 \%$ & $332 \%$ & $285 \%$ & $1000 \%(\mathrm{n}=1016)$ \\
\hline Suecin & $59 \%$ & $173 \%$ & $31,4 \%$ & $455 \%$ & $100,0 \%(n=979)$ \\
\hline Fintandin & $173 \%$ & $33.6 \%$ & $265 \%$ & $22,6 \%$ & $100,0 \%(n=1000)$ \\
\hline Suim & $70 \%$ & $223 \%$ & $302 \%$ & $40,6 \%$ & $100,0 \%(n=1223)$ \\
\hline Alemarin & $380 \%$ & $360 \%$ & $165 \%$ & $95 \%$ & $1000 \%(n=1991)$ \\
\hline Total & $23,0 \%$ & $29,1 \%$ & $263 \%$ & $216 \%$ & $100,0 \%(n=11343)$ \\
\hline
\end{tabular}

Fuente: ASEP, Encuesta Mundial de Valores 2005-2008. Elaboración propia. en los diez países analizados, pueden explicarse por diferencias en los niveles de renta (a pesar de que se trata de países de elevado nivel de desarrollo). Al tratar de relacionar el grado de expresividad de cada país (sumando el porcentaje de respuestas de mixtosexpresivos y expresivos) con variables relacionadas con los niveles de renta (PIB per capita ) o de desarrollo (Índice de Desarrollo Humano) no se han encontrado relaciones estadísticamente signifi cativas. Sin embargo, al considerar el impacto del nivel de estudios (medido como el porcentaje de las personas de cada país que señalan tener alguna formación universitaria) sí se encuentra una clara ordenación del nivel de expresividad de los países (Gráfi co 1).

Gráfi co 1. Relación entre la expresividad en las orientaciones laborales y el nivel de estudios en 10 paises europeos

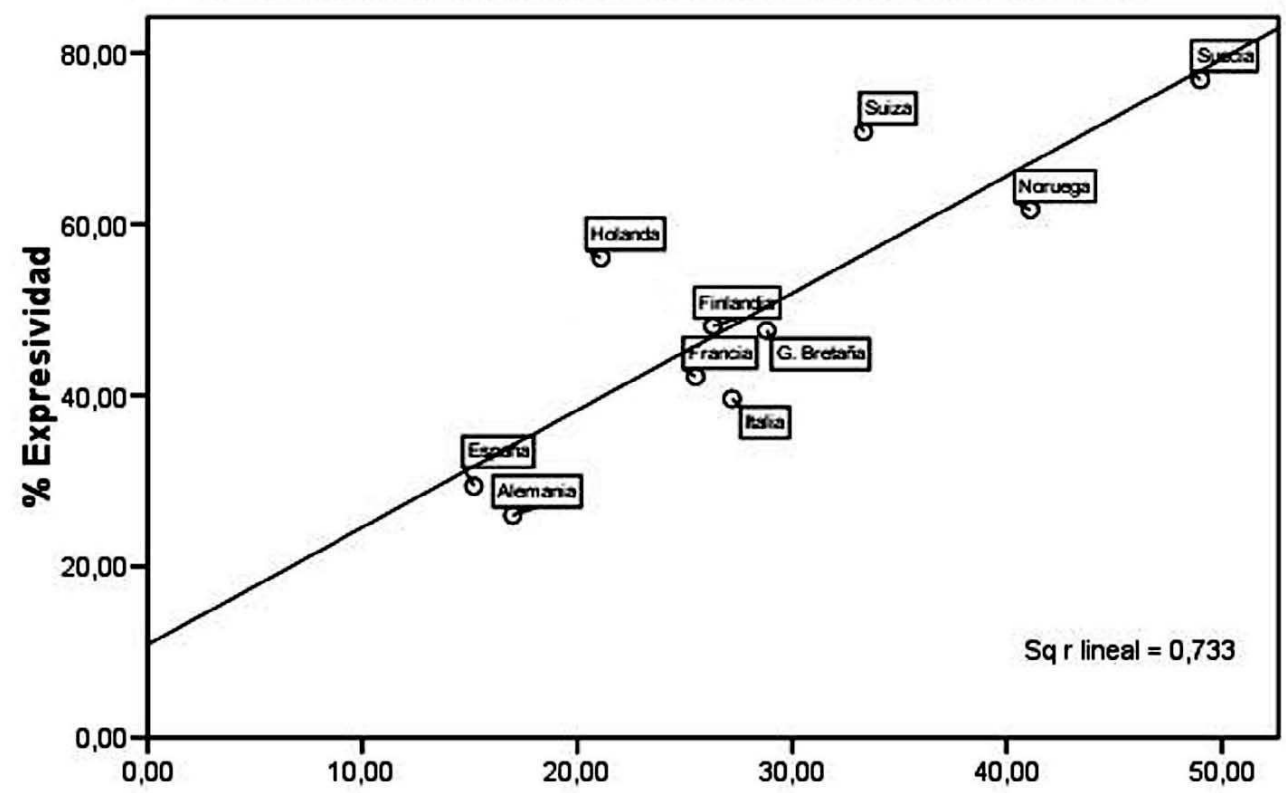

\% DE POBLACIÓN CON NIVEL ESTUdios ALTO

Fuente: ASEP, Encuesta Mundial de Valores 2005-2008. Elaboración propia.

Además en la tabla inicial de correlaciones (tabla 1) se observan muchas asociaciones entre las variables explicativas, como era previsible. El nivel de estudios está relacionado 
con todas las otras variables explicativas -en especial con el nivel de ingresos y la ocupación-. Por ello se han calculado las correlaciones parciales entre el nivel de estudios y las orientaciones laborales, controlando por el resto de las variables explicativas (tabla 5) y se comprueba que el efecto del nivel de estudios se mantiene en todos los casos prácticamente con la misma intensidad.

Por otra parte, se observa que existen diferencias significativas entre el país de realización de la encuesta y prácticamente todas las variables explicativas (la única excepción es la variable sexo). Dichas diferencias refl ejan la existencia de composiciones muestrales diferentes, posiblemente debidas a que las poblaciones de origen también tienen composiciones diferentes en cuanto a las variables explicativas. Por esta razón se han obtenido las correlaciones de las orientaciones laborales y las variables explicativas en cada uno de los países analizados, que se presentan a continuación.

Tabla 5. Correlaciones entre las orientaciones laborales y el nivel de estudios para una muestra de diez países europeos, controlando por distintas variables explicativas

\begin{tabular}{|c|c|}
\hline Controlando por: & Correlación parciale entre las orientaciones labales y elnivel de estutios \\
\hline Cohortes por mino de nacimiento & $0,294^{+40,000011254}$ \\
\hline Oomación & $0,285^{+40,000} 10784$ \\
\hline Escaln de ingresos & $0,262+0,0009799$ \\
\hline 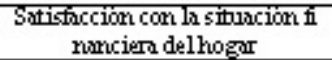 & $0,264^{4+0,00011191}$ \\
\hline Sinuación laboral & $0286 * 0,00011197$ \\
\hline Seso & $0,289 * 40,00011254$ \\
\hline Pás & $0,270^{+4} 0,00011254$ \\
\hline
\end{tabular}

Fuente: ASEP, Encuesta Mundial de Valores 2005-2008. Elaboración propia.

a En cada casilla de la tabla se muestra en primer lugar la correlación parcial, en segundo lugar el nivel de significación y en tercer lugar el tamaño de la muestra (excluidos los casos de no respuesta) ** La correlación es signifı cativa al nivel 0.01 (bilateral)

En Francia, Gran Bretaña, España, Finlandia y Alemania, el nivel de estudios tiene una mayor correlación con las orientaciones laborales que ninguna de las otras variables (Tabla 6). En Italia las orientaciones laborales están ligeramente más relacionadas con la ocupación que con el nivel de estudios. En Holanda el mejor predictor de las orientaciones laborales es la satisfacción con la situación financiera del hogar. En Noruega, Suecia y Suiza, el sexo muestra una elevada correlación con las orientaciones laborales, si bien en los tres casos el siguiente predictor en importancia es el nivel de estudios. 
Tabla 6: Correlaciones entre las orientaciones laborales y distintas variables explicativas ${ }^{\mathrm{a}}$ en cada uno de los diez países europeos

\begin{tabular}{|c|c|c|c|c|c|c|c|}
\hline & $\begin{array}{l}\text { Hinolde } \\
\text { oitribioi }\end{array}$ & 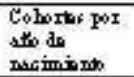 & Qcmsion & $\begin{array}{c}\text { Erish de } \\
\text { ingerior }\end{array}$ & 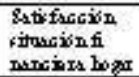 & $\begin{array}{l}\text { Sirancion } \\
\text { leborsl }\end{array}$ & 20000 \\
\hline Fisteris & $\begin{array}{l}0,207^{+4} \\
0,000994\end{array}$ & $\begin{array}{l}-0,0370,240 \\
965\end{array}$ & $\begin{array}{l}-0,176 \% 4 \\
0,000965\end{array}$ & $\begin{array}{c}0,175 \% 6 \\
0,000873\end{array}$ & $9,096+0,003$ & $\begin{array}{l}0,0970,1+6 \\
978\end{array}$ & $\begin{array}{c}0,063 \\
0,172978\end{array}$ \\
\hline Eran & $\begin{array}{l}0,131 \text { की } \\
0,00010+8\end{array}$ & $\begin{array}{l}-0,07200,018 \\
1062\end{array}$ & $\begin{array}{c}-0,109+6 \\
0,0001039\end{array}$ & $\begin{array}{c}0,0360,195 \\
840\end{array}$ & $\begin{array}{l}0,073+0,011 \\
1053\end{array}$ & $\begin{array}{l}0,11564 \\
0,009967\end{array}$ & $\begin{array}{c}0,196 \% 4 \\
0,000987\end{array}$ \\
\hline Itstis & $\begin{array}{l}0,116+6 \\
0,000990\end{array}$ & $\begin{array}{l}0,083+0,0,007 \\
992\end{array}$ & $\begin{array}{l}-0,26064 \\
0,000 \mathrm{n}\end{array}$ & $\begin{array}{c}0,19566 \\
0,000663\end{array}$ & $\begin{array}{l}0,198 \times 0,0,000 \\
963\end{array}$ & $\begin{array}{l}0,113^{\circ} \\
0,013944\end{array}$ & $\begin{array}{c}0,104^{4} \\
0,013992\end{array}$ \\
\hline Holands & $\begin{array}{l}0,095 \% 6 \\
0,003980\end{array}$ & $\begin{array}{l}-0,090 \%+4 \\
0,005994\end{array}$ & $\begin{array}{c}-0,0340,306 \\
937\end{array}$ & $\begin{array}{l}0,105 \text { क6 } \\
0,000+771\end{array}$ & $\begin{array}{l}0,150+0,0000 \\
974\end{array}$ & $\begin{array}{l}0,115^{\circ} \\
0,010962\end{array}$ & $\begin{array}{c}0,121+4 \\
0,002992\end{array}$ \\
\hline $\mathrm{E}_{p} \mathrm{pl}_{\mathrm{k}}$ & $\begin{array}{l}0,248+6 \\
0,0001164\end{array}$ & $\begin{array}{l}0,111 \text { का } \\
118000\end{array}$ & ..11\& & $\begin{array}{c}0,097+6 \\
0,0011097\end{array}$ & $\begin{array}{l}0,07060,016 \\
1176\end{array}$ & $\begin{array}{l}0,1177^{60} \\
0,0011179\end{array}$ & $\begin{array}{c}0,040 \\
0,5921185\end{array}$ \\
\hline Horw ga & $\begin{array}{l}0,162 * 4 \\
0,0001014\end{array}$ & $\begin{array}{l}0,0600,057 \\
1016\end{array}$ & $\begin{array}{l}-0,09646 \\
0,003967\end{array}$ & $\begin{array}{c}0,117^{46} \\
0,000935\end{array}$ & $\begin{array}{l}0,118^{*+0,000} \\
101+\end{array}$ & $\begin{array}{l}0,0790,408 \\
1014\end{array}$ & $\begin{array}{r}0,17864 \\
0,0001016\end{array}$ \\
\hline 8 owcin & $0,1+1 * 4$ & 96860,617 & $\begin{array}{c}-0,0610,059 \\
956\end{array}$ & $\begin{array}{c}-0,0080,819 \\
921\end{array}$ & $\begin{array}{l}0,0280,390) \\
965\end{array}$ & $\begin{array}{l}0,110^{\circ} \\
0,0259 \pi 7\end{array}$ & $\begin{array}{c}0,189^{* 4} \\
0,000979\end{array}$ \\
\hline Fmlandis & $\begin{array}{l}0,189+6 \\
0,00010+2\end{array}$ & $\begin{array}{l}0,0290,349 \\
1043\end{array}$ & $\begin{array}{c}-0,08164 \\
0,0091034\end{array}$ & $\begin{array}{c}0,072^{\circ} \\
0,026956\end{array}$ & $\begin{array}{l}0,07600,014 \\
1043\end{array}$ & $0,110^{\circ}$ & $\begin{array}{c}0,0 \pi 1 \\
0,1 \leqslant 81001\end{array}$ \\
\hline $8 \sin 2$ & $\begin{array}{l}0,163^{+\infty} \\
0,0001021\end{array}$ & $\begin{array}{l}0,0160,400 \\
1027\end{array}$ &. .1017 & $\begin{array}{c}0,117^{60} \\
0,000909\end{array}$ & $\begin{array}{l}0,062+0,048 \\
1026\end{array}$ & $\begin{array}{l}0,127^{+6} \\
0,0001211\end{array}$ & $\begin{array}{c}0,168+4 \\
0,0001214\end{array}$ \\
\hline Abmantin & $\begin{array}{l}0,2916 \\
0,0002016\end{array}$ & $\begin{array}{l}-0,0130,555 \\
2033\end{array}$ & $\begin{array}{c}-0,187 \% 6 \\
0,0001935\end{array}$ & $\begin{array}{c}0,137+4 \\
0,0001821\end{array}$ & $\begin{array}{l}0,116+00,000 \\
1003\end{array}$ & $\begin{array}{l}0,1177^{4} \\
0,0001951\end{array}$ & $\begin{array}{c}0,043 \\
0,1961992\end{array}$ \\
\hline
\end{tabular}

Fuente: ASEP, Encuesta Mundial de Valores 2005-2008. Elaboración propia. Nota: véase tabla 1

** La correlación es signifi cativa al nivel 0.01 (bilateral)

* La correlación es signifi cativa al nivel 0.05 (bilateral)

Tabla 7: Correlaciones parciales entre las orientaciones laborales y el nivel de estudios, controlando por distintas variables explicativas ${ }^{a}$ en cada uno de los diez países europeos

\begin{tabular}{|c|c|c|c|c|c|c|}
\hline & $\begin{array}{l}\text { Cohortes por } \\
\text { rifo de } \\
\text { nacimiento }\end{array}$ & Ocupación & $\begin{array}{l}\text { Escala de } \\
\text { ingresos }\end{array}$ & $\begin{array}{l}\text { Satiaficción } \\
\text { sinuación fi } \\
\text { narcier } \\
\text { hoger }\end{array}$ & $\begin{array}{l}\text { Sinuación } \\
\text { laboral }\end{array}$ & Sexo \\
\hline \multirow{2}{*}{ Francin } & $0,251^{\circ}$ & $0,188^{*}$ & $0,172^{\circ}$ & $0209 * 4$ & $0,226^{\circ}$ & $0,219+0,000$ \\
\hline & 0,000974 & 0,000974 & 0,000864 & 0,000970 & 0,000974 & 974 \\
\hline Gram & $0,244^{4 *}$ & $0,207^{\circ+}$ & $0,204^{6 *}$ & $0217^{64}$ & $0,234^{\circ}+$ & $0,238^{+*} 0,000$ \\
\hline Bretsiñ & 0,000970 & 0,000947 & 0,000772 & 0,000962 & 0,000970 & 970 \\
\hline \multirow[b]{2}{*}{ Itslix } & $0,194^{\circ 4}$ & $0,077^{\circ}$ & $0,152^{64}$ & $0,192 \%$ & $0,211^{\circ}$ & $0,211^{\circ}+0,000$ \\
\hline & 0,000977 & 0,038714 & 0,000653 & 0,000969 & 0,000969 & 977 \\
\hline \multirow{2}{*}{ Holmin } & $0,135^{\circ 6}$ & $0,112^{6}$ & $0,125^{\circ 6}$ & $0,099+4$ & $0,122^{46}$ & $0,119+0,000$ \\
\hline & 0,000986 & 0,001943 & 0,000775 & 0,002977 & 0,000974 & 986 \\
\hline \multirow{2}{*}{ Españ } & $0,232+6$ & & $0,233^{64}$ & $0,240^{\circ 6}$ & $0,257^{64}$ & $0,258+0,000$ \\
\hline & 0,0001162 & 0 & 0,0001078 & 0,0001154 & 0,0001161 & 1162 \\
\hline \multirow{2}{*}{ Nonueg } & $0,131^{\star *}$ & $0,121^{* *}$ & $0,106^{+4}$ & $0,131^{4}$ & $0,139^{\circ}$ & $0,145+0,000$ \\
\hline & 0,0001011 & 0,000984 & 0,001932 & 0,0001009 & 0,0001010 & 1011 \\
\hline \multirow{2}{*}{ Suecin } & $0,142^{\circ+}$ & $0,138^{\circ}+$ & $0,150^{\circ}+$ & $0,142^{* *}$ & $0,148^{* *}$ & $0,142+0,000$ \\
\hline & 0,000968 & 0,000955 & 0,000919 & 0,000965 & 0,000967 & 968 \\
\hline \multirow{2}{*}{ Finlandir } & $0,189+4$ & $0,174^{\circ+}$ & $0,178^{\circ}+$ & $0,183^{64}$ & $0,191^{\circ}$ & $0,182^{+}+0,000$ \\
\hline & 0,000996 & 0,000988 & 0,000913 & 0,000996 & 0,000996 & 996 \\
\hline \multirow{2}{*}{ Suim } & $0,167^{\circ}$ & & $0,133^{\circ}$ & $0,167^{64}$ & $0,167^{\circ 4}$ & $0,183^{\circ}+0,000$ \\
\hline & 0,0001214 & 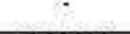 & 0,0001096 & 0,0001213 & 0,0001213 & 1214 \\
\hline \multirow{2}{*}{ Alemarix } & $0312^{4 *}$ & $0295^{\circ 6}$ & $0,278^{\circ}$ & $0297^{\circ 6}$ & $0,325+6$ & $0,308^{\circ} 0,000$ \\
\hline & 0,0001971 & 0,0001877 & 0,0001770 & 0,0001949 & 0,0001938 & 1971 \\
\hline
\end{tabular}

Fuente: ASEP, Encuesta Mundial de Valores 2005-2008. Elaboración propia. Notaa: véase tabla 1.

** La correlación es signifi cativa al nivel 0.01 (bilateral)

* La correlación es signifi cativa al nivel 0.05 (bilateral)

48 Ahora bien la única variable explicativa que correlaciona signifi cativamente con las orientaciones laborales en la dirección esperada en todos los países, sin excepción, es el nivel de estudios. Además se puede comprobar cómo en todos los países dichas correlaciones se mantienen significativas, con el mismo signo y prácticamente con la misma intensidad, incluso cuando se controla por el resto de las variables explicativas (Tabla 7).

49 En cuanto a las cohortes por año de nacimiento sólo ofrecen una correlación significativa con las orientaciones laborales en España, Holanda, Italia y Gran Bretaña y 
en ninguno de los casos muestran mayor intensidad con las orientaciones laborales que el nivel de estudios (en Holanda están casi al mismo nivel aunque, como ya se ha dicho más arriba, en este país el mejor predictor de las orientaciones laborales es la satisfacción con la situación fi nanciera del hogar). Además, tanto en Gran Bretaña como en Holanda el signo de la asociación es contrario al esperado teóricamente (la relación negativa de las cohortes y las orientaciones laborales que implica que los nacidos en las últimas cohortes son menos expresivos). En España y en Italia la dirección de las relaciones indica que las cohortes más recientes son más expresivas. ${ }^{4}$

La ocupación es una variable con correlaciones significativas en seis de los países analizados -las excepciones son Holanda y Suecia, donde la correlación no es significativa-; por otra parte, en España y Suiza no se dispone de información sobre esta variable. No obstante, en aquellos países en los que sí se halla una relación significativa entre la ocupación y las orientaciones laborales, la intensidad de dicha relación es menor que la que se obtiene con el nivel de estudios, salvo en el caso de Italia.

El nivel de ingresos tiene una relación significativa con las orientaciones laborales en todos los países excepto en Gran Bretaña y Suecia, aunque con menor intensidad que el nivel de estudios, excepto en Holanda.

La satisfacción con la situación financiera del hogar presenta relaciones significativas con las orientaciones laborales en prácticamente todos los países, salvo Suecia, aunque en algunos, como Gran Bretaña, España, Finlandia y Suiza, la relación es débil.

La situación laboral permite establecer diferencias significativas en las orientaciones laborales en la mayoría de los países; las excepciones son Francia y Noruega, si bien en Italia, Holanda, Suecia y Finlandia las asociaciones son bastante débiles. Además, la situación laboral no aparece en ningún caso como el mejor predictor de las orientaciones laborales.

La variable sexo está relacionada significativamente con las orientaciones laborales en seis de los países analizados, especialmente en Suecia y Noruega, donde supera a otras variables explicativas. Los países en los que el sexo no ayuda a diferenciar las orientaciones laborales son Francia, España, Finlandia y Alemania.

\section{Conclusiones}

Los hallazgos obtenidos muestran que la variable nivel de estudios resulta ser el factor más determinante de la demanda de recompensas expresivas del trabajo. Esta capacidad predictora del nivel de estudios se ha demostrado tanto en el nivel individual (micro) como en el nacional o por países (macro). Ello convierte al nivel de estudios como el mejor predictor en la difusión y desarrollo de los valores expresivos en el trabajo.

Las razones por las cuales el nivel de estudios es el principal determinante de la difusión de las orientaciones expresivas en el trabajo son variadas, pero teóricamente pueden apuntarse principalmente dos: por un lado la fuerte inversión en dinero y tiempo que supone la educación superior probablemente tendrá un impacto sobre las aspiraciones laborales que se situarán más allá del salario y la estabilidad; por otro lado la educación superior supone una exposición más prolongada a un adiestramiento orientado al desarrollo personal (self-orientation) y al logro de metas individuales. 
Por otra parte, las diferencias observadas entre países en el impacto del nivel de estudios sobre las orientaciones laborales pueden ser atribuidas a diferencias en los sistemas educativos. El análisis de estas diferencias en los sistemas educativos es algo que escapa al propósito de este artículo. Pero, en cualquier caso, es de destacar que en todos los países el nivel de estudios está correlacionado con las orientaciones expresivas en el trabajo.

Sin embargo, no debe darse por garantizada una supuesta evolución hacia los valores expresivos en el trabajo, como supone la hipótesis del cambio generacional, que no se ha mostrado consistente con los datos. Es más, aunque se incrementen en el futuro los niveles de estudios entre la población, ello no sería suficiente para garantizar un incremento de los valores expresivos, porque se ha demostrado que cuando hay una fuerte demanda de recompensas expresivas en el trabajo que no pueden ser satisfechas, se incrementarán las demandas de recompensas instrumentales (materialistas) como mecanismo psicológico de compensación y defensa (Alderfer, 1972).

\section{BIBLIOGRAFÍA}

ABRAMSON, Paul R. \& INGLEHART, Ronald (1992), “Generational Replacement and Value Change in Eight West European Societies”, British Journal of Political Science, Vol. 22, 183-228.

ABU-SAAD, Ismael (1997), “Gender as a Determinant of Work Values among University Students in Israel”, Journal of Social Psychology, Vol. 137, 749-763.

ALDERFER, Clayton. P. (1972), Existence, Relatedness and Growth: Human Needs in Organizational Settings, New York, The Free Press. ASEP: http://www.jdsurvey.net/jds/jdsurvey.jsp

AYERBE, M. (2000), “El trabajo” en: Orizo, F. A. y Elzo, J. (Dirs.) in España 2000, entre el localismo y la globalidad. La Encuesta Europea de Valores en su tercera aplicación, 1981-1999, Madrid, Universidad de Deusto. Fundación Santa María, 157-179.

CHERRINGTO, David J. (1980), The Work Ethic. Working Values and Values that Work, New York, Amacom.

DEX, Shirley. (1988), Women's Attitudes towards Work, New York, St. Martin's Press.

DUCH, Raymond. M. \& TAYLOR, Michaell. A. (1993), "Postmaterialism and the Economic Condition”, American Journal of Political Science, Vol. 37, No. 3, 747-779.

DUCH, Raymond. M. \& TAYLOR, Michaell. A. (1994), “A Replay to Abramson and Inglehart's Education, Security, and Postmaterialism”, American Journal of Political Science, Vol. 38, No. 3, 815-825.

GOLDTHORPE, John H., LOCKWOOD, David, BECHHOFER, Frank \& PLATT, Jennifer (1968), The Affluent Worker: Industrial Attitudes and Behaviour, Cambridge, Cambridge University Press.

HARDING, Stephen D. \& HIKSPOORS, F. J. (1995), “New Work Values: in Theory and in Practice”, International Journal of Social Sciences, Vol. 47, No. 145, 441-455.

INGLEHART, Ronald (1991), El cambio cultural en las sociedades industriales avanzadas, Madrid, Centro de Investigaciones Sociológicas. 
INGLEHART, Ronald (1998), Modernización y posmodernización. El cambio cultural, económico y político en 43 sociedades, Madrid, Centro de Investigaciones Sociológicas.

INGLEHART, Ronald. y WELZEL, Christian. (2006): Modernización, cambio cultural y democracia: la secuencia del desarrollo humano, Madrid, Centro de Investigaciones Sociológicas.

MASLOW, Abraham. H. (1954), Motivation and personality, New York, Harper and Row.

MOW International Research Team (1987), The Meaning of Working, London, Academic Press.

MUÑOZ GOY, Celia (2005), Las orientaciones laborales en España y sus determinantes sociodemográfi cos, Santiago de Compostela, Universidad de Santiago de Compostela, Servicio de Publicaciones e Intercambio Científico.

PINO ARTACHO, Juan del y BERICAT ALASTUEY, Eduardo (1996), Valores sociales en la cultura andaluza: encuesta mundial de valores, Andalucía 1996, Madrid, Centro de Investigaciones Sociológicas.

ROKEACH, Milton (1973), The Nature of the Human Values, New York, The Free Press.

ROS, Maria, SCHWARTZ, Shalom H. \& SURKISS, Shoshana (1999), "Basic Individual Values, Work Values, and the Meaning of Work”, Applied Psychology, Vol. 48, No. 1, 49-71.

ROWE, Reba \& SNIZEK, William E. (1995), “Gender Differences in Work Values. Perpetuating the Myth”, Work and Occupations, Vol. 22, No. 2, 215-229.

RUSSELL, H. (1998), “The Rewards of Work”, Roger Jowell, R.; Curtice, J.; Park, A.; Brook, L.; Thomson, K. y Bryson, C. (Eds.), British -and European- Social Attitudes. How Britain Differs. The 15th Report. Social and Community Planning Research, Adershot, England, Ashgate Publishing Limited, 77-100.

SVERKO, Branimir. \& SUPER, Donald E. (1995), “The Findings of the Work Importance Study", Super, D. E. y Sverko, B. (Eds.), Life Roles, Values, and Careers. International Findings of the Work Importance Study, San Francisco, Jossey-Bass, 349-358.

VAUS, David \& MCALLISTER, Ian (1991), “Gender and Work Orientation”, Work and Occupations, Vol. 18, No. 1, 72-93

VEIRA VEIRA, José Luis Y MUÑOZ GOY, Celia (2004), "Valores y actitudes hacia el trabajo en Europa Occidental”, Revista Española de Sociología, No. 4, 51-66

YANKELOVICH, Daniel, ZETTERBERG, Hans, STRUMPEL, Burkhard. \& SHANKS, Michael (1985), The World at Work. An International Report on Jobs, Productivity and Human Values, New York, Octagon Books

YANKELOVICH, Daniel. (1994), “How Changes in the Economy are Reshaping American Values”, in Aaron, H. J.; Mann, T. E. y Taylor, T., Values and Public Policy, Washington, Brookings Books

ZANDERS, Harry (1994), “Changing Work Values”, in Ester, P., Halman, L. y De Moor, R. (Eds.), The Individualizing Society. Value Change in Europe and North America, Tilburg, The Netherlands, Tilburg University Press, 129-153.

\section{NOTAS}

1. La diferencia entre necesidades primarias y secundarias se basa en la conocida teoría de la jerarquía de las necesidades de Maslow (1954), de modo que las necesidades de tipo primario engloban las necesidades fisiológicas y de seguridad, mientras que las de tipo secundario se corresponden con las necesidades sociales o de afi liación, las de estima y las de autorrealización. 
2. Las fechas de realización del trabajo de campo y los respectivos tamaños muestrales fueron: Alemania, 2006 n=2064; España, 2007 n=1213; Finlandia, 2005, n= 1016; Francia, 2006 n= 1001; Gran Bretaña, 2006 n= 1041; Italia, 2005, n=657; Noruega, 2007, n=1665; Países Bajos, 2006, n=1050; Suecia, 2006, n= 1003; Suiza, 2007 n= 1241.

3. El hecho de que la medida de asociación no sea significativa no quiere decir exactamente que las orientaciones laborales de jóvenes y mayores sean iguales sino que no hay un orden que permita afi rmar si la relación es de tipo creciente o decreciente. En realidad, si se analiza la tabla de contingencia entre las orientaciones laborales y la cohorte por año de nacimiento se observa que hay una cierta relación entre dichas variables, pero no se observa que las cohortes más jóvenes sean más expresivas que las cohortes que corresponden a las personas de mayor edad sino que la relación tiene una forma curvilínea, pues precisamente los más jóvenes y los de más edad presentan mayores porcentajes de orientaciones instrumentales, mientras que las cohortes intermedias se inclinan ligeramente en mayor medida por las orientaciones expresivas. Además, cabe destacar que se ha explorado esta relación considerando sólo la población ocupada y se aprecia que sí existe una relación significativa entre las orientaciones laborales y la cohorte por año de nacimiento, pero el signo del coeficiente de asociación es contrario al que cabría esperar desde el punto de vista teórico -de la evolución/cambio de los valores por reemplazo generacional-, pues el signo negativo indica que entre las primeras cohortes (que se corresponde con personas de más edad) hay una mayor demanda de recompensas expresivas que entre las últimas cohortes (que se corresponden con las personas más jóvenes).

4. Sin embargo, se han analizado de modo exploratorio estas relaciones considerando sólo la población ocupada, en cuyo caso la asociación entre las orientaciones laborales y las cohortes por año de nacimiento desaparece tanto en Italia como en España -lo que puede deberse a que se ha reducido la muestra en el sentido de que desaparecen las cohortes de edad más extremas y por la relación que existe entre la situación laboral y las cohortes de edad-, mientras que en Gran Bretaña y Holanda se mantienen aunque más débiles que en el caso de la población total- y con el mismo signo.

\section{RESÚMENES}

El objetivo de este artículo es verificar dos proposiciones teóricas que tratan de explicar las variaciones observadas en la distribución de los valores instrumentales y expresivos del trabajo. Por un lado la hipótesis del cambio intergeneracional, que supone que un cambio de valores es el resultado del reemplazo de las generaciones más viejas por las más jóvenes y, por otro lado, la hipótesis que afirma que el cambio está determinado por factores relacionados con el status socioeconómico del individuo. Los datos de diez países europeos muestran que la hipótesis del cambio intergeneracional es inconsistente y refuerzan la importancia del nivel de estudios como factor explicativo de las orientaciones laborales.

The aim of this paper is to test two theoretical propositions that try to explain the observed variations in the distribution of instrumental and expressive work values. On the one hand the generation effect hypothesis, which assumes that a value shift is the result of the replacement of older generations by younger ones, and, on the other hand, the hypothesis that states that the change is determined by factors related to individual socioeconomic status. Data, from ten European countries, show that the hypothesis of generational change is inconsistent and reinforce the importance of educational level as a factor to explain work orientations.

L'objectif de cet article est de vérifier deux propositions théoriques qui essaient d'expliquer les variations observées dans la distribution des valeurs instrumentales et expressives du travail. D'un côté l'hypothèse du changement intergénérationnel, qui suppose qu'un changement de valeurs soit le résultat du remplacement des plus vieilles générations par les plus jeunes et, d'un 
autre côté, l'hypothèse qui affirme que le changement est déterminé par des facteurs relatifs au statut socio-économique de l'individu. Les données de dix pays européens montrent que l'hypothèse du changement intergénérationnel est inconsistante et renforcent l'importance du niveau d'études comme facteur explicatif des orientations de travail.

ÍNDICE

Mots-clés: travail, valeurs, materialisme et postmaterialisme, génération, éducation

Palabras claves: trabajo, valores, materialismo y posmaterialismo, generación, educación

Keywords: work, values, materialism and post materialism, generation, education

\section{AUTORES}

\section{JOSÉ LUIS VEIRA VEIRA}

Facultad de Sociología, Universidade da Corunha veiraj@udc.es

\section{CELIA MUÑOZ GOY}

Departamento de Sociología, Universidade da Corunha munozg@udc.es 